Enferm Bras 2020;19(4Supl);S13-S20

https://doi.org/10.33233/eb.v19i4.4299

\title{
RELATO DE EXPERIÊNCIA \\ Profissional residente no enfrentamento da COVID-19: relato de experiência no contexto da enfermagem intensiva
}

Raquel Margarida Silva Freire*, Gyuliana Santana Batista*, Talita de Araújo de Carvalho*, Douglas de Souza e Silva**, Tássia Nery Faustino**, Magno Conceição das Merces, D.Sc. ${ }^{\star \star \star *}$

${ }^{*}$ Enfermeira, Programa de Residência Multiprofissional em Terapia Intensiva, Universidade do Estado da Bahia/Departamento Ciências da Vida, Salvador/BA, Brasil, ${ }^{* \star E}$ Enfermeiro, Mestrando do Programa de Pós-graduação em Ciências da Saúde. Universidade Federal da Bahia, Salvador/BA, ${ }^{\star * \star}$ Enfermeira, Doutoranda do Programa de Pós-Graduação em Medicina e Saúde, Universidade do Estado da Bahia/Departamento Ciências da Vida Salvador/BA Brasil, ${ }^{* * * \star}$ Enfermeiro, Universidade do Estado da Bahia/Departamento Ciências da Vida, Salvador, Bahia, Brasil

Recebido em 24 de julho de 2020; aceito em 14 de agosto de 2020.

Correspondência: Raquel Margarida Silva Freire, 1ํTravessa Jorge Amado, 09, Imbuí, 41720110 Salvador BA

Raquel Margarida Silva Freire: rfreire00@gmail.com

Gyuliana Santana Batista: gyulibatista@gmail.com.

Talita de Araújo de Carvalho: araujotalita383@gmail.com

Douglas de Souza e Silva: douglasss-gbi@hotmail.com

Tássia Nery Faustino: tfaustino@uneb.br

Magno Conceição das Merces: mmerces@uneb.br

\section{Resumo}

Objetivo: Descrever a experiência de profissionais residentes no enfrentamento da COVID-19 no contexto da enfermagem intensiva. Métodos: Trata-se de um estudo do tipo relato de experiência vivenciado por três profissionais residentes em saúde no enfrentamento da COVID-19 no contexto da enfermagem intensiva em dois hospitais situados na região metropolitana de Salvador, no estado da Bahia, no período de março a maio de 2020. Resultados: Constatou-se mudanças nos fluxos operacionais dos serviços em saúde, o racionamento dos equipamentos de proteção individual dentro das instituições hospitalares e como essas demandas impactam na saúde mental dessas profissionais que estão na linha de frente na assistência a esses pacientes. Conclusão: A pandemia causada pelo SARS-COV-2 coloca em discussão a estrutura das unidades de terapia intensiva existentes no país, sendo primordial a capacitação técnica e científica dos profissionais de saúde, garantia no fornecimento dos equipamentos de proteção individual e suporte psicológico a esses trabalhadores.

Palavras-chave: infecções por coronavírus, pandemias, profissionais de enfermagem, saúde do trabalhador, atenção à saúde.

\footnotetext{
Abstract

Resident professional facing COVID-19: experience report in the context of intensive nursing

Objective: To describe the experience of professionals residing in coping with COVID-19 in the context of intensive nursing. Methods: This is a study report type of experience lived by three health professionals in coping with COVID-19 in the context of intensive nursing in two hospitals located in the metropolitan region of Salvador, in the state of Bahia, from March to May 2020. Results: There were changes in the operational flows of health services, the rationing of personal protective equipment within hospital institutions and how these demands impact on the mental health of these professionals who are at the forefront in assisting these patients. Conclusion: The pandemic caused by SARS-VOC-2 puts into question the structure of the intensive care units existing in the country, being essential the technical and scientific training of health professionals,
} 
guarantee in the supply of personal protective equipment and psychological support to these workers.

Keywords: coronavirus infections, pandemics, nurse practitioners, occupational health, health care.

\section{Resumen \\ Profesional de la salud residente frente al COVID-19: relato de experiencia en el contexto de enfermería intensiva}

Objetivo: Describir la experiencia de los profesionales residentes en el manejo de COVID-19 en el contexto de la enfermería intensiva. Métodos: Se trata de un tipo de relato de experiencia vivida por tres profesionales de la salud residentes frente al COVID-19 en el contexto de la enfermería intensiva en dos hospitales ubicados en la región metropolitana de Salvador, en el estado de Bahía, de marzo hasta mayo de 2020. Resultados: Se constató que hubo cambios en los flujos operativos de los servicios de salud, el racionamiento de los equipos de protección personal dentro de las instituciones hospitalarias y cómo estas demandas impactan en la salud mental de estos profesionales que están a la vanguardia en la asistencia a estos pacientes. Conclusión: La pandemia causada por el SARS-VOC-2 cuestiona la estructura de las unidades de cuidados intensivos existentes en el país, siendo esencial la capacitación técnica y científica de los profesionales de la salud, la garantía en el suministro de equipos de protección personal y apoyo psicológico a estos trabajadores

Palabras-clave: infecciones por coronavirus, pandemias, enfermeras practicantes, salud laboral, atención a la salud.

Introdução

A doença respiratória da COVID-19 causada pelo novo coronavírus (SARS-COV-2) foi caracterizada pela Organização Mundial da Saúde (OMS) no mês de março como uma pandemia devido a sua rápida dispersão entre os continentes e a sua situação de agravamento. Seu surgimento se deu na cidade de Wuhan, província de Hubei, na China, e vem atingindo o globo vertiginosamente, desencadeando uma emergência em Saúde Pública de importância internacional [1-2].

O espectro clínico de um indivíduo infectado pela COVID-19 é variado e pode evoluir desde a forma assintomática até casos graves. Sua letalidade é baixa, em torno de $3 \%$, porém sua transmissibilidade é alta [3-4], principalmente em locais fechados e ambientes hospitalares que podem ser favorecidos por contato próximo, sem proteção, com secreções por meio de gotículas salivares e aerossóis [5].

O vírus pode levar ao desenvolvimento de sintomas leves como a de um resfriado, com presença de tosse, febre, coriza, dor de garganta e dispneia até manifestações clínicas mais graves que evoluem para Síndrome Respiratória Aguda Grave (SRAG), com o desenvolvimento de insuficiência respiratória com necessidade de suporte ventilatório mecânico, assim como para disfunção aguda de múltiplos órgãos [6], o que requer uma assistência de saúde mais complexa ofertada em uma Unidade de Terapia Intensiva (UTI).

No contexto da pandemia mundial, muitos profissionais de saúde estão com suas rotinas de trabalhos modificadas devido à emergência nos cuidados aos infectados e na contenção da disseminação do vírus [7]. A equipe de enfermagem, a qual integram enfermeiros, técnicos e auxiliares de enfermagem, representa a maior porcentagem da força de trabalho no campo da saúde com seus cuidados ininterruptos à beira do leito, que abrangem desde os mais básicos aos mais complexos [8].

O cuidado de enfermagem dentro do sistema de saúde é um componente fundamental dentro do cenário de pandemia que o mundo vem vivenciando. No Brasil, é possível contabilizar mais de dois milhões de profissionais [9] que atuam incansavelmente em diferentes setores e contextos sociais para identificar as necessidades de cuidado da população e atuar em suas diferentes dimensões.

O enfermeiro apropria-se de um papel decisivo e nesse momento precisa avaliar suas competências, atitudes e habilidades para a oferta de um cuidado seguro para paciente, profissional e equipe, o que requer treinamentos e adaptações diárias através de protocolos e fluxos institucionais que ocasionam mudanças nas rotinas dos serviços de saúde [10].

Nesse contexto, destaca-se o papel do profissional enfermeiro residente em saúde que atua dentro do programa de Residência Multiprofissional em Saúde regulamentada pela Lei Federal ํo 11,129 de 30 de junho de 2005, na modalidade de pós-graduação lato sensu, voltada 
para a educação em serviço. Esse profissional é inserido no campo de serviço e atua juntamente com a equipe de saúde com perfil capaz de transformar as práticas tradicionais com base no entendimento de uma nova cultura e intervenção em saúde [11].

O profissional residente em saúde está presente nos serviços e compartilha dessa abrupta mudança de rotina que ocasiona ansiedade, medo e angústia frente ao vírus que se propaga de forma acelerada e promove intensas mudanças frente a uma estrutura de saúde que se torna insuficiente para o melhor atendimento dos pacientes infectados e que pode entrar em colapso a qualquer momento. É necessário adaptação ao cenário atual, mudanças de fluxos operacionais dentro dos serviços, utilização de equipamentos de proteção individual (EPI) de forma racionada e acima de tudo preservar a saúde mental desse profissional, para que 0 trabalho seja realizado de forma eficiente e que não provoque adoecimentos.

Dessa forma, o objetivo deste estudo foi descrever a experiência de profissionais residentes em saúde no enfrentamento da COVID-19 no contexto da enfermagem intensiva.

Trata-se de um estudo do tipo relato de experiência vivenciado por profissionais residentes em saúde no enfrentamento da COVID-19 no contexto da enfermagem intensiva em dois hospitais situados na região metropolitana de Salvador, no estado da Bahia, no período de março a maio de 2020. Um dos hospitais da região não é referência para a COVID-19, porém como a infecção alcançou o estágio de transmissão comunitária, todos os casos que adentram a instituição são considerados suspeitos e seguem-se as recomendações do Ministério da Saúde. O outro hospital é referência e possui leitos específicos para o tratamento desses pacientes.

O lócus da ação está baseado no cotidiano de três profissionais enfermeiras residentes, sendo duas do primeiro ano e uma do segundo, que estão vivenciando e acompanhando através das mudanças de fluxos nos serviços de saúde o cuidado a pacientes suspeitos e confirmados para COVID-19, assim como o racionamento e, por vezes, escassez dos equipamentos de proteção individual dentro das instituições, e como todas essas alterações afetam a saúde mental dessas profissionais e quais são as estratégias utilizadas para o enfrentamento dessa pandemia.

Os dados que foram relatados também representam a observação acerca do comportamento das instituições frente à pandemia do coronavírus. Foram feitas buscas na Biblioteca Virtual em Saúde (BVS) com os seguintes descritores: "Infecção por coronavírus", "Pandemia" "Profissionais de enfermagem", "Saúde do trabalhador" e "Atenção hospitalar" com o operador booleano AND que nos auxiliou na busca de artigos que nos direcionaram na discussão acerca das experiências relatadas. Dados do Ministério da Saúde e do Conselho Federal de Enfermagem também foram utilizados.

\section{Resultados e discussão}

As pandemias virais emergentes exigem à saúde pública demandas peculiares que sobrecarregam o sistema de saúde e alteram seus fluxos de atendimentos. Os gestores, frente a um vírus de rápida propagação que pode levar a graves impactos nas instituições, atuam no planejamento e na preparação de protocolos e fluxogramas que atendam às demandas impostas por esse cenário da COVID-19 e que afeta de forma abrupta a rotina dos profissionais de saúde [12].

Estudos em países já acometidos pelo novo coronavírus direcionaram a organização dos atendimentos nas instituições hospitalares brasileiras [13]. A Declaração de Consenso do Comitê de Supervisão das Diretrizes do American College of Chest Physicians (CHEST) apresenta 22 sugestões que podem ser adotadas durante a logística de uma pandemia por administradores no planejamento hospitalar aos pacientes graves, incluindo requisitos de equipamentos, suprimentos, preparação e organização de pessoal, que são elementos que fornecem capacidade para que o cuidado nesse momento crítico seja garantido [14].

Nesse contexto, em que os fluxos operacionais são mudados, é possível perceber na prática clínica o bombardeamento de informações que chegam para os trabalhadores de saúde que necessitam adequar suas habilidades no manejo ao paciente acometido pela COVID-19. Com proporção global, pesquisadores de vários países estudam sobre o vírus e é possível que as informações sejam lançadas quase que instantaneamente, influenciando diretamente na forma como esses pacientes serão conduzidos [15].

Um estudo realizado na China caracterizou as manifestações clínicas de 138 pacientes acometidos pelo coronavírus [16] e em outro estudo descritivo realizado no mesmo país, foram 
identificadas as características epidemiológicas de 99 pacientes concluindo que pacientes acima dos 55 anos e com comorbidades prévias podiam desenvolver doenças respiratórias graves e até fatais, como a Síndrome do Desconforto Respiratório Agudo [17], facilitando que essas informações fossem divulgadas, assimiladas por outros países com acometimento e difundindose para conhecimento e treinamento dos profissionais de saúde que trabalham constantemente no reconhecimento e direcionamento da COVID-19.

Conforme protocolos do Ministério da Saúde e levando em consideração experiências de vários países, pacientes que apresentam sintomatologia leve da síndrome gripal são orientados pelos profissionais a manterem 0 isolamento social domiciliar mantendo-se em distanciamento social com adoção de medidas de precaução para evitar novas contaminações. Pacientes que adentram o hospital com sintomas de dispneia, febre e que possam apresentar complicações respiratórias, necessitam de vigilância e são internados em leitos específicos para essa enfermidade [10-18].

Com esse cenário epidemiológico e com as medidas de isolamento social que orientam a população a permanecer em casa, principalmente se apresentarem sintomas leves da doença, fez com que muitos indivíduos acometidos por outras emergências permanecessem em suas casas, o que é preocupante, pois em um estudo realizado na França que objetivou avaliar os efeitos das medidas de contenção da população, foi constatado uma queda drástica de internações referentes a doenças cardiovasculares por conta das medidas de isolamento social que tornam esses pacientes susceptíveis a graves consequências [19].

Com isso, observou-se redução na procura por atendimento médico na instituição hospitalar de referência observada neste estudo, fazendo com que setores específicos durante a pandemia da COVID-19 tenham baixa nos serviços oferecidos como, por exemplo, Centro Cirúrgico (CC), Central de Material de Esterilização (CME), Hemodinâmica, Pediatria, ambulatórios, tornando os profissionais desses setores sujeitos a remanejamentos para suprir a necessidade do serviço diante do cenário atual. As equipes desses serviços encontram- se em constante revezamento para atuar nas UTIs específicas da COVID-19, gerando incertezas na rotina desse profissional, sendo perceptível o desconforto que essas mudanças têm causado aos mesmos, principalmente porque a prática clínica dentro das UTIs é diferente dos outros setores e requer habilidade técnica e científica para atuar nesse contexto.

Constata-se que a assistência prestada nas UTIs coorte para COVID-19 objetiva a correção das disfunções orgânicas agudas ocasionadas pelo SARS-COV-2, o que exige cuidados complexos que devem ser prestados pela equipe multiprofissional evitando procedimentos que promovam a disseminação de aerossóis e a contaminação do ambiente e desses profissionais [20]. Com isso, os profissionais precisam de atualizações diárias e é perceptível o nível de exaustão causada por esse novo quadro desafiador para todos.

Dessa forma, observou-se no hospital referência para COVID-19 a realização de treinamentos e capacitações pela Comissão de Controle de Infecção Hospitalar $(\mathrm{CClH})$, voltados para toda a equipe multiprofissional, na qual foram efetuadas orientações acerca da adequada paramentação e desparamentação por meio da técnica de simulação realística, assim como, distribuição de EPIs, como óculos de proteção e sapatos de material impermeável. Foram disponibilizados cartazes em toda extensão da UTI sobre a forma correta de paramentação e desparamentação e as enfermeiras referências dessas unidades estão supervisionando e encorajando a equipe para o seguimento dos passos instituídos.

Durante o treinamento, também foram expostos alguns procedimentos de geração de aerossóis (PGAs), conforme orientação do Ministério da Saúde, como: Intubação Orotraquel (IOT), aspiração de vias aéreas, nebulização, broncoscopia, endoscopia, utilização da bolsa válvula - máscara na ressuscitação cardiopulmonar (RCP), porém essas informações foram pontuais e sem demonstração de como os profissionais poderiam atuar nessas situações, de forma a reduzir/eliminar a aerossolização.

O que é observado no hospital que não é referência no atendimento da COVID 19, mas que pode assistir pacientes suspeitos da doença, é que as duas UTIs se diferenciam por ter somente quatro leitos para o cuidado a esses pacientes, que são leitos de isolamentos. A equipe também foi treinada pela $\mathrm{CClH}$, porém as capacitações foram pontuais e deficientes.

Profissionais de enfermagem que trabalham nesse hospital também atuam em outros serviços de referência para a COVID-19, estando submetidos à elevada carga horária de trabalho, com rotina assistencial e administrativa exaustivas. Os serviços têm mantido o gerenciamento habitual do cuidado através do preenchimento de várias escalas, impressos, diagnósticos e prescrições de enfermagem, projeto terapêutico e acompanhamento de visita multiprofissional, não revendo e priorizando as atividades administrativas consideradas essenciais, diante do 
cenário de sobrecarga de trabalho decorrente da assistência ao paciente crítico com a doença. Associado a isso, nesse cenário de pandemia, procedimentos complexos têm sido efetuados diariamente nos pacientes com a doença, como diálise contínua e posicionamento prona. Em relação a este último, o seu emprego está associado à redução da mortalidade em pacientes em ventilação mecânica [21], contudo as equipes não tiveram treinamento de como efetuá-lo, recorrendo à busca individual de materiais acerca da temática em seus dias de folga, para prestar uma assistência segura e de qualidade.

Com essas múltiplas demandas assistenciais e administrativas, observa-se que: muitos profissionais da área da enfermagem intensiva têm solicitado demissão; número elevado de afastamentos por suspeita ou confirmação de contaminação pelo vírus e ocorrência de faltas nos plantões por crise de pânico diante do cenário imposto, o que gera medo, ansiedade e insegurança, reduzindo o quadro de profissionais. Diante disso, os serviços têm aumentado o número de contratações de profissionais para suprir os déficits decorrentes dos afastamentos, contudo, por vezes, estes não possuem formação/experiência no atendimento ao paciente grave, comprometendo assim a sua própria segurança e a do paciente.

$\mathrm{O}$ aceleramento da pandemia no Brasil tem ocasionado à falta de leitos e equipamentos necessários para o cuidado que impactam diretamente na saúde dos trabalhadores. É possível observar um cenário de preocupação quantos aos EPIs, decorrente do aumento exponencial do seu uso nos serviços. A escassez desses materiais já é uma realidade em vários países [22], o que resulta em sofrimento constante para os trabalhadores diante do risco de contaminação, principalmente porque a China é nossa grande produtora de materiais hospitalares.

O estágio de transmissão comunitária do SARS-COV-2 gera incerteza no atendimento aos pacientes sem diagnóstico, contribuindo para o aumento na utilização dos materiais de proteção. Com isso, como medida de contenção do uso de EPIs, os hospitais passaram a realizar o registro diário para a aquisição desses equipamentos e o monitoramento do seu uso, fato que é observado na nossa prática e que pode ser corroborada por um estudo descritivo realizado em Porto Alegre, em que medidas de controle e racionamento para evitar a falta destes nos hospitais estão sendo adotadas [23].

Outro fator observado gerador de ansiedade é o risco aos quais os trabalhadores de saúde estão expostos na paramentação e desparamentação ao atender um paciente acometido pela COVID-19. A utilização desses materiais de forma ininterrupta gera um desconforto com o seu uso prolongado, tendo o profissional que se expor a longas jornadas de trabalho sem descanso, assim como alimentação e hidratação prejudicados, visto que para atender a essas necessidades básicas teriam que se expor à retirada frequente dos EPIs, que aumenta o risco de contaminação. Devido a isso, alguns profissionais estão trabalhando com fraldas higiênicas e mulheres estão iniciando o uso de hormônios para evitar o ciclo menstrual.

Em um estudo do tipo Survey realizado no Reino Unido que objetivou conhecer a opinião dos profissionais de saúde e suas percepções sobre as estratégias no gerenciamento da pandemia, revelou 1007 respostas nas quais constataram que $27,8 \%$ dos entrevistados relataram não se sentir confiantes frente ao atendimento ao paciente infectado pela COVID-19, $66 \%$ consideravam que não havia equipamento de proteção individual disponível satisfatoriamente e $52,1 \%$ consideraram que não foi fornecido treinamento suficiente para a equipe que está na linha de frente desse atendimento [24], corroborando assim nossas observações práticas dentro das instituições hospitalares.

A contaminação e o adoecimento já se tornam uma realidade, como observado no estudo realizado na China em que 3.387 profissionais de saúde foram infectados pela COVID-19 com 22 mortes registradas [25]. No Brasil, pelos dados do Conselho Federal de Enfermagem até o dia 27 de maio de 2020, foram identificados 157 óbitos de profissionais enfermeiros/técnicos/auxiliares de enfermagem que padeceram pelo acometimento do vírus, tornando nosso país o maior em mortes de profissionais de saúde [26]. Os profissionais estão em constante estresse, exacerbado pelas inúmeras incertezas impostas pela situação atual vivida, exposição contínua nos seus locais de trabalho, medo do adoecimento, frustração com as perdas diárias de pacientes, salários baixos e isolamento da família que impactam diretamente na qualidade de vida e no desempenho do seu trabalho.

Nessa conjuntura, o profissional enfermeiro residente acompanha junto à equipe de saúde os entraves e as lutas diárias das rotinas impostas pela COVID-19, observando as mudanças nos fluxos operacionais dos hospitais em que desenvolvem as atividades de estágio/trabalho, sendo submetidos ao racionamento dos equipamentos de proteção e se questionam sobre seu papel como profissional e ser humano frente à pandemia do novo coronavírus. Com esse novo cenário lidam com o medo de não serem amparados numa possível 
contaminação com a exposição diária na sua prática laboral, dentro das unidades de terapia intensiva.

Na vigência de uma pandemia os profissionais de saúde são colocados à prova com a intensificação de medidas no combate ao agente patogênico para evitar sua propagação e infecção à população, com isso, uma mistura de emoções que trazem medo e aumentam os níveis de ansiedade e estresse em indivíduos ditos como saudáveis, podem desencadear transtornos psiquiátricos pré-existentes [27].

Nos referidos hospitais, os residentes multiprofissionais em terapia intensiva trabalham no primeiro ano de sua especialização com dois pacientes e no segundo ano com quatro pacientes graves, o que se torna desafiador frente a uma pandemia. Essa situação afeta exponencialmente os residentes do primeiro ano que vivenciam a UTI neste momento difícil em que estão iniciando a formação no atendimento ao paciente crítico, conhecendo as tecnologias, patologias e principais procedimentos efetuados nesses indivíduos, a tempo em que experimentam mudanças que a sobrecarregam, pois vivenciam o prejuízo nas preceptorias do serviço já que os enfermeiros assistenciais estão sobrecarregados. Associado a isso, precisam se ajustar ao contexto imposto, necessitando sair da casa de seus pais, pertencentes ao grupo de risco, para evitar a contaminação dos mesmos.

Ser residente do primeiro ano nesta fase de pandemia se torna desafiador visto que 0 residente ainda se encontra em fase de adaptação à terapia intensiva, um ambiente que exige atuação direta a pacientes com diferentes condições clínicas, diversos micro-organismos e formas de contágio, lidando com o desconhecido, tendo que se atualizar ao mesmo tempo em que se expõem diariamente a situações diversas.

A preceptoria da residência se torna imprescindível neste âmbito de mudanças, o ponto de apoio dos residentes, principalmente para os residentes do primeiro ano que iniciam suas jornadas dentro da terapia intensiva, um setor complexo, novo para muitos que não o vivenciaram em suas graduações. Aliado a isso, o residente se depara com a urgência em adquirir novos conhecimentos para aprimorar a sua assistência e com a problemática de lidar com suas próprias dificuldades no dia a dia e enfrentamento do abalo psicológico causado pela pandemia e fatores externos.

O aumento dos fatores de risco para sofrimento psíquico dos profissionais da área da saúde se tornam cada vez mais visíveis: medo se de ser infectado, adoecer e morrer; possibilidade de contaminar seus familiares; exposição à morte em larga escala e a não vivência do luto por colegas de profissão/conhecidos; distanciamento dos entes queridos; atenção extrema e constante nas técnicas de trabalho; além do aumento da carga de trabalho devido ao afastamento de profissionais com COVID-19, torna essa realidade difícil de ser vivenciada.

Desta forma, são necessárias intervenções para evitar que os estressores da pandemia causem impactos na saúde mental dos profissionais de saúde. O equilíbrio na saúde mental dos profissionais residentes é indispensável para que a assistência prestada seja de forma eficaz e segura. Destaca-se que no cenário atual, os profissionais que já sofram com distúrbios psicológicos podem ter o seu agravamento pela atual pandemia do medo [28].

Conclusão

A pandemia causada pelo SARS-COV-2 coloca em discussão a estrutura das unidades de terapia intensiva existentes no país, que para se adequar ao novo contexto epidemiológico, tiveram que modificar seus fluxos operacionais para abarcar a necessidade de saúde que se apresentava, o que torna primordial a capacitação técnica e científica dos profissionais de saúde que estão na linha de frente. Também se faz necessário garantir equipamentos de proteção individual para uma assistência segura, assim como apoio psicológico e elaboração de um plano de cuidados para que exposições contínuas aos estressores da pandemia não tornem os trabalhadores adoecidos e vulneráveis a acometimentos psicológicos graves.

\section{Referências}

1. Word Health Organization. Considerations for quarantine of individuals in the context of containment for coronavirus disease (COVID-19): Interim guidance [Internet]. Geneva; 2020 [citado 2020 Mai 20]. Disponível em:

https://apps.who.int/iris/handle/10665/331299

2. Chang Le, Yan Y, Wang L. Coronavirus disease 2019: Coronaviruses and blood safety. Transfus Med Rev 2020; 34(2):75-80. https://doi.org/10.1016/j.tmrv.2020.02.003 
3. Zhu N, Zhang D, Wang W, Xingwang Li, Yang B, Song J et al. A novel Coronavirus from patients with pneumonia in China, 2019. N Engl J Med 2020;382(8):727-33. https://doi.org/10.1056/NEJMoa2001017

4. Centers for Disease Control and Prevention (CDC). Interim U.S. guidance for risk assessment and public health management of healthcare personnel with potential exposure in a healthcare setting to patients with Coronavirus Disease (COVID-19). 2020 [citado 2020 Mai 22]. Disponível em: https://www.cdc.gov/coronavirus/2019ncov/hcp/guidance-risk-assesment-hcp.html

5. Li R, Pei S, Chen B, Song Y, Zhang T, Yang W et al. Substantial undocumented infection facilitates the rapid dissemination of novel coronavirus (SARS-CoV2). Science 368(6490):489-93. https://doi.org/10.1126/science.abb3221

6. Vincent JL, Taccone FS. Understanding pathways to death in patients with COVID-19. Lancet Respir Med 2020;8(5):430-32. https://doi.org/10.1016/S2213-2600(20)30165-X

7. Souza LPS, Souza AG. Enfermagem brasileira na linha de frente contra o novo Coronavírus: quem cuidará de quem cuida? J Nurs Health 2020;10(n.esp.):e20104005. [citado 2020 Mai 23]. Disponível em: https://periodicos.ufpel.edu.br/ojs2/index.php/enfermagem/article/view/18444/11240

8. Souza RR. A enfermagem oncológica no enfrentamento da pandemia de COVID-19: Reflexões e recomendações para a prática de cuidado em oncologia. Rev Bras Cancerol 2020;66. https://doi.org/10.32635/2176-9745.RBC.2020v66nTemaAtual.1007

9. Conselho Federal de Enfermagem [Internet]. Brasília, DF: Cofen; 2020 [citado 2020 Mai 25]. Enfermagem em números. Disponível em: http://www.cofen.gov.br/enfermagemem-numeros

10. Silva ACR, Jesus TS, Santos SS, Santos GJ. COVID-19, o novo coronavírus: um alerta emergencial para as principais estratégias de prevenção da saúde pública. Scire Salutis 2020;10(2):56-63. https://doi.org/10.6008/CBPC2236-9600.2020.002.0004

11. Lei $n^{\circ} 11.129$, de 30 de junho de 2005. Presidência da República. Casa Civil. Subchefia para Assuntos Jurídicos. Institui a Residência em Área Profissional de Saúde e cria a Comissão Nacional de Residência Multiprofissional em Saúde CNRMS. Diário Oficial da União. [Internet] 2005 [citado 2020 Mai 27]. Disponível em: http://www.planalto.gov.br/ccivil 03/ Ato20042006/2005/Lei/L11129.htm

12. Centers for Disease Control and Prevention. Planning and preparedness resources. Washington, DC: CDC; 2020. [citado 2020 Maio 28]. Disponível em: https://www.cdc.gov/coronavirus/2019-ncov/php/pandemic-preparednessresources.html

13. Kumar A, Gupta, PK, Srivastava A. A review of modern technologies for tackling COVID-19 pandemic. Diabetes Metab Syndr 2020;14:569-73. https://doi.org/10.1016/j.dsx.2020.05.008

14. Einav S, Hick JL, Hanfling D, Devereaux AV, Christian MD. Surge capacity logistics: care of the critically ill and injured during pandemics and disasters: CHEST consensus statement. Chest 2014;146(4):e17S-e43S. https://doi.org/10.1378/chest.14-0734

15. Phua J, Weng L, Ling L, Egi M, Lim CM, Divatia JV et al. Intensive care management of coronavirus disease 2019 (COVID-19): challenges and recommendations. Lancet Respir Med 2020;8:506-17. https://doi.org/10.1016/S2213-2600(20)30161-2

16. Wang D, Hu B, Hu C, Zhu F, Liu X, Zhang J et al. Clinical characteristics of 138 hospitalized patients with 2019 novel coronavirus-infected pneumonia in Wuhan, China. Jama 2020;323(11):1061-69. Disponível em: https://jamanetwork.com/journals/jama/article-abstract/2761044

17. Chen N, Zhou M, Dong X, Qu J, Gong F, Han Y et al. Epidemiological and clinical characteristics of 99 cases of 2019 novel coronavirus pneumonia in Wuhan, China: a descriptive study. The Lancet 2020;395(10223):507-13. https://doi.org/10.1016/S01406736(20)30211-7

18. Huang C, Wang Y, Li X, Ren L, Zhao J, Hu Y et al. Clinical features of patients infected with 2019 novel coronavirus in Wuhan, China. The Lancet 2020;395(10223):497-506. https://doi.org/10.1016/S0140-6736(20)30183-5

19. Huet F, Prieur C, Schurtz G, Gerbaud E, Silberman SM, Vanzetto G et al. One train may hide another: Acute cardiovascular diseases could be neglected because of the COVID-19 pandemic. Arch Cardiovasc Dis 2020;113:303-7. https://doi.org/10.1016/i.acvd.2020.04.002 
20. Qiu H, Tong Z, Ma P, Hu M, Peng Z, Wu W et al. Intensive care during the coronavirus epidemic. Intensive Care Med 2020;46:576-8. https://doi.org/10.1007/s00134-02005966-y

21. Alhazzani W, Moller MH, Arabi YM, Loeb M, Gong MN, Oczkowski EFS et al. Surviving sepsis campaign: guidelines on the management of critically ill adults with coronavirus disease 2019 (COVID-19). Intensive Care Med 2020;46:854-87. https://doi.org/10.1007/s00134-020-06022-5

22. Ranney ML, Griffeth V, Jha, AK. Critical supply shortages-the need for ventilators and personal protective equipment during the COVID-19 pandemic. N Engl J Med 2020;382(18):e41. https://doi.org/10.1056/NEJMp2006141

23. Rodrigues NH, Silva LG. Gestão da pandemia coronavírus em um hospital: relato de experiência profissional. J Nurs Health 2020;10(n.esp.):e20104004. Disponível em: https://periodicos.ufpel.edu.br/ojs2/index.php/enfermagem/article/view/18530

24. Iqbal MR, Chaudhuri A. COVID-19: Results of a national survey of United Kingdom healthcare professionals' perceptions of current management strategy-a crosssectional questionnaire study. Int J Surg 2020;79:156-61. https://doi.org/10.1016/.i.jsu.2020.05.042

25. Wang J, Zhou M, Liu F. Exploring the reasons for healthcare workers infected with novel coronavirus disease 2019 (COVID-19) in China. J Hosp Infect 2020. https://doi.org/10.1016/i.jhin.2020.03.002

26. Conselho Federal de Enfermagem, Brasília, DF: Cofen; 2020. Brasil é o país com mais mortes de enfermeiros por COVID-19 no mundo. [citado 2020 Jun 02]. Disponível em: http://www.cofen.gov.br/brasil-e-o-pais-com-mais-mortes-de-enfermeiros-por-COVID19-no-mundo-dizem entidades 80181.html

27. Shigemura J, Ursano RJ, Morganstein JC, Kurosawa M, Benedek DM et al. Public responses to the novel 2019 coronavirus (2019-nCoV) in Japan: mental health consequences and target populations. Psychiatry Clin Neurosci 2020;74(4):281-2. https://doi.org/10.1111/pcn.12988.

28. Silva D, Pimentel R, Merces M. COVID-19 and the pandemic of fear: reflections on mental health. Rev Saúde Pública 2020;54:46. https://doi.org/10.11606/s1518$\underline{8787.2020054002486}$ 